\title{
Incorporación de criterios eco-hidrológicos en la gestión forestal: adaptación a la escasez de agua de una masa marginal de encina
}

\author{
González-Sanchis, M. ${ }^{*}$, del Campo, A², Lidón, A², Lull, C², Bautista, I. ${ }^{2}$, García- \\ Prats, A. ${ }^{2}$, Francés, F. ${ }^{3}$ \\ ${ }^{1}$ Departamento de Ingeniería hidráulica y medio ambiente, Universitat Politècnica de València, Valencia, España. \\ ${ }^{2}$ Departamento de Ingeniería hidráulica y medio ambiente, Grupo de investigación en ciencia forestal \\ y tecnología (Re-ForeST), Universitat Politècnica de València, Valencia, España.
}

${ }^{3}$ Instituto Universitario de Investigación de Ingeniería del Agua y Medio Ambiente (IIAMA), Universitat Polècnica de València, Valencia, España

*e-mail: macgonsa@gmail.com

\section{Resumen}

El Manejo Forestal Adaptativo (MFA) en entornos semiáridos tiene como objetivo adaptar el bosque a la escasez de agua mediante una regulación artificial de la estructura y la densidad del mismo. En el presente trabajo se aplica el MFA a una masa marginal de Quercus ilex con el fin de optimizar los recursos hídricos y tratando de evitar a su vez el posible impacto negativo derivado de una pérdida excesiva de suelo y nutrientes. La masa se encuentra en la Cuenca Mediterránea, dentro del Monte Público de La Hunde, Valencia (E de España), donde dos parcelas contiguas, control y tratamiento, de $1800 \mathrm{~m}^{2}$ cada una, fueron establecidas. La orientación (NO), pendiente (30 \%) y densidad (861 árboles por ha) fueron las mismas en ambas parcelas. La parcela tratamiento fue aclarada en Mayo del 2012, reduciendo la densidad de 861 a 414 árboles por ha. La parcela control no fue aclarada. Los efectos del aclareo en los ciclos hidrológico y biogeoquímico fueron caracterizados en ambas parcelas y comparados entre sí. Los resultados indicaron un efecto temprano del aclareo que optimiza el ciclo hidrológico. Se encontró un incremento significativo de la transpiración, escorrentía cortical y humedad del suelo en la parcela tratada. Por el contrario, no se observaron efectos ni en la escorrentía superficial ni en la trascolación. De la misma forma, el MFA parece no afectar significativamente al ciclo biogeoquímico del bosque, ya que no se encontraron diferencias significativas en el contenido de $\mathrm{C}, \mathrm{N}$ y $\mathrm{P}$ en el agua de ambas parcelas. La removilización de nutrientes parece verse afectada por el resalveo. La parcela tratada muestra una menor eficiencia en el uso de los nutrientes, probablemente derivada de una mayor disponibilidad de agua. Con estos resultados, podemos afirmar que la aplicación de un adecuado MFA posibilita la optimización del ciclo hidrológico sin dañar significativamente el ciclo de los nutrientes. De la misma forma, además de la satisfacción de las necesidades hídricas y nutricionales del ecosistema, el MFA incrementa la disponibilidad de agua para el resto de la cuenca.

Palabras clave: Manejo Forestal Adaptativo, ciclo hidrológico, semiárido, ciclo biogeoquímico. 


\section{Introducción}

La disponibilidad de agua en la Cuenca del Mediterráneo es escasa, y depende principalmente de la escorrentía producida en las zonas montañosas (Ives et al., 1997; Viviroli y Weingartner, 2004; de Jong et al., 2009). En general, las zonas montañosas suponen entre un 20 y un $50 \%$ del caudal total de la cuenca; sin embargo, en zonas semiáridas tales como el Mediterráneo, dicho aporte puede suponer entre un 50 y un $90 \%$ del total (Viviroli y Weingartner, 2004). Desafortunadamente, la cuenca Mediterránea ha sido identificada como una de las áreas más vulnerables al cambio global (Giorgi, 2006), donde se esperan mayores incrementos de la temperatura y la aridez que en otras regiones (Cubasch et al., 1996). Además, las montañas pertenecientes a la cuenca Mediterránea también se ven afectadas por cambios en la demanda de agua por actividades humanas así como por modificaciones en la cobertura vegetal y usos del suelo. Dichas modificaciones afectan: i) al balance hídrico debido a sus efectos en la evapotranspiración, en los ratios de interceptación (Joffre y Rambal, 1993), en la dinámica de la humedad del suelo (Correia, 1999), y en la recarga de acuíferos (Callegari et al., 2003). Por tanto, el mantenimiento de la cantidad y calidad de la escorrentía en las zonas montañosas a través del manejo de dichos entornos constituye un reto importante con el que afrontar los problemas de escasez de agua en el Mediterráneo (Messerli et al., 2004).

Por tanto, el Manejo Forestal Adaptativo (MFA) en el Mediterráneo trataría de incrementar la escorrentía o la recarga de acuíferos así como mejorar la sostenibilidad del bosque. Sin embargo, este MFA podría también ocasionar una pérdida de nutrientes y suelo, así como incrementar la evaporación del agua del suelo. Por ello, el MFA a aplicar debe de encontrar un compromiso que satisfaga tanto las necesidades del bosque como las de la cuenca en la que se integra. En el presente trabajo se aplica el MFA a una masa marginal de Quercus ilex con el fin de optimizar los recursos hídricos, pero tratando de evitar a su vez el posible impacto negativo derivado de una pérdida excesiva de suelo y nutrientes.

\section{Material y métodos}

El área de estudio, Monte Público de La Hunde y Palomeras (3950’30" N, $1^{\circ} 12^{\prime} 30^{\prime \prime}$ O), se encuentra en el Suroeste de la provincia de Valencia, a $950 \mathrm{~m}$ sobre el nivel del mar. El clima es Mediterráneo con una precipitación media anual de 466 mm y una temperatura media anual de $13.7^{\circ} \mathrm{C}$. Los suelos presentan un elevado contenido en carbonato (26-38 \%; $\mathrm{pH} 7.7-8.2)$, con una profundidad media aproximada de $50-60 \mathrm{~cm}$ y una textura areno-arcillosa. Las parcelas experimentales forman parte de un típico carrascal Mediterráneo, de unos 60 años de edad aproximadamente, dominado por Quercus ilex ssp rotundifolia, en el que también encontramos algunos pies de Quercus faginea y Pinus halepensis. La densidad arbórea es de 861 árboles ha ${ }^{-1} \mathrm{y}$ su estrato arbustivo algo escaso, en el que predominan el Juniperus phoenicea y el Juniperus oxycedrus. 


\subsection{Diseño experimental:}

Se han seleccionado dos parcelas experimentales contiguas (Control y Tratamiento), de idénticas orientación (NO), pendiente $(30 \%)$ y extensión $\left(1800 \mathrm{~m}^{2}\right)$. Tan sólo en una de las parcelas se ha aplicado el tratamiento selvícola pertinente (resalveo), dejando la otra parcela como testigo. El tratamiento fue efectuado en Abril del 2012, siguiendo dos criterios: apertura de la masa y eliminación de los pies sometidos, favoreciendo siempre a las especies $Q$. ilex y $Q$. faginea, las más adelantadas en la sucesión natural. Como resultado, la cubierta forestal se disminuyó de 861 a 414 árboles $\mathrm{ha}^{-1}$. El tratamiento fue llevado a cabo bajo la supervisión del Servicio Forestal público de Valencia. La madera gruesa fue extraída, mientras que la fina fue triturada y reincorporada a la parcela Tratamiento.

Las parcelas fueron inventariadas en Marzo del 2012 siguiendo las variables de estructura forestal: Índice de Área Foliar $\left(\mathrm{LAI}, \mathrm{m}^{2} \mathrm{~m}^{-2}\right)$, área basimétrica $\left(\mathrm{BA}, \mathrm{m}^{2}\right.$ ha${ }^{1}$ ), densidad de árboles ( $\mathrm{D}$, árbol ha ${ }^{-1}$ ) y clase diamétrica (ver Tab. 1).

Tabla 1. Estructura forestal en las parcelas control y tratamiento. D: densidad arbórea (árboles/ ha). BA: Área Basimétrica $\left(\mathrm{m}^{2} \mathrm{ha}^{-1}\right)$. LAI: Índice de área foliar $\left(\mathrm{m}^{2} \mathrm{~m}^{-2}\right)$.

\begin{tabular}{|c|c|c|}
\hline Parámetro & Control & Tratamiento \\
\hline D & 861 & 414 \\
\hline BA & 11.4 & 9.1 \\
\hline LAI & 1.8 & 1.1 \\
\hline
\end{tabular}

E1 LAI fue estimado mediante un sensor LAI-2000 (LI-COR, 1991). Las lecturas fueron tomadas bajo radiación solar directa según Molina and Del Campo (2011 y 2012). El área basimétrica y la densidad fueron estimadas mediante la medida de los diámetros y el conteo de todos los árboles de la parcela. La distribución diamétrica de la masa fue obtenida midiendo los diámetros tan sólo de los pies de Q. ilex como especie objetivo del presente estudio.

Tabla 2. Inventario inicial de la parcela Control.

\begin{tabular}{|c|c|c|c|}
\hline Clases diamétricas & Diámetro $(\mathrm{cm})$ & $\mathrm{N}^{\circ}$ Árboles & Árbol/ha \\
\hline I & $<7.5$ & 74 & 205.6 \\
\hline II & $7.5-11$ & 58 & 161.1 \\
\hline III & $11-15$ & 71 & 197.2 \\
\hline IV & $>15$ & 56 & 155.6 \\
\hline
\end{tabular}

\subsection{Caracterización de los ciclos hidrológico y biogeoquímico:}

Las variables que nos permiten caracterizar el ciclo hidrológico en una masa forestal son: precipitación neta, trascolación, escorrentía cortical, humedad del suelo, 
escorrentía superficial y transpiración del arbolado. Todas ellas fueron recolectadas durante el año hidrológico 2012-2013.

La precipitación se midió en un área abierta a $20 \mathrm{~m}$ de las parcelas mediante un pluviómetro (Davis, USA) programado para medir a intervalos de tiempo de $10 \mathrm{mi}$ nutos.

Para medir la trascolación se instalaron en cada parcela 3 filas de 3 canalones paralelos a la pendiente, de $1 \mathrm{~m}^{2}$ cada uno. Cada fila de canalones se conectó a un pluviómetro (Davis, USA) programado para medir a intervalos de tiempo de 10 minutos.

La escorrentía cortical se midió en 4 árboles de cada parcela, representantes de cada una de las 4 clases diamétricas obtenidas en el inventario inicial. Se instaló en cada árbol un anillo de poliuretano sellado con silicona y conectado a un pluviómetro (Davis, USA) programado para medir a intervalos de tiempo de 10 minutos.

La transpiración del arbolado se medió también en 4 árboles por parcela mediante sensores de flujo de savia, representantes de cada una de las 4 clases diamétricas obtenidas en el inventario inicial (ver Tab. 2). En cada árbol se instaló una sonda de velocidad de flujo de savia (HRM-30, ICT International Pty Ltd., Armidale,Australia) a $20 \mathrm{~cm}$ de la base, en la parte superior de la pendiente y con orientación Sur.

La humedad del suelo se registró cada media hora, mediante sondas de humedad y temperatura ( 5 TE, Decagon ${ }^{\circledR}$, USA) situadas en 3 puntos por parcela y a 3 profundidades: 5,15 y $30 \mathrm{~cm}$.

La escorrentía superficial se caracterizó mediante la instalación, en cada parcela, de dos zanjas de recogida de agua al final de la pendiente, de $4 \mathrm{~m}^{2}$ cada una. Las zanjas fueron conectadas a un pluviómetro (Davis, USA) programado para medir a intervalos de tiempo de 10 minutos.

El ciclo biogeoquímico fue caracterizado a través del análisis del contenido de $\mathrm{N}$, P y C en la lluvia, escorrentía cortical, trascolación, escorrentía superficial y en el lixiviado. La removilización de nutrientes de la carrasca en ambas parcelas fue caracterizada mediante el análisis y comparación del contenido en C, N, K y P en los brotes, hojas, tallos y hojarasca de la misma.

La comparación entre parcelas se llevó a cabo a través del análisis de la t-student mediante el programa $\mathrm{R}$.

\section{Resultados}

\subsection{Ciclo hidrológico}

La precipitación total durante el año hidrológico 2013-2013 fue de $587.3 \mathrm{~mm}$, distribuidos en 115 eventos de lluvia. La distribución de dicha precipitación en ambas parcelas no mostró apenas diferencias significativas. La interceptación y la escorrentía superficial fueron igual en las parcelas tratamiento y control (ver Tabla 3). Por el contrario, se observó un incremento del consumo individual $\left(\mathrm{mm}^{2}\right.$ ábol $\left.^{-1} \mathrm{dí}^{-1}\right)$ de agua por parte del estrato arbóreo. Sin embargo, dicho consumo individual llevado a tér- 
minos globales de la parcela $\left(\mathrm{mm} \mathrm{m}^{-2}\right.$ día $\left.^{-1}\right)$, fue significativamente $(\mathrm{p} \leq 0.05)$ menor en la parcela tratamiento que en la control. Como consecuencia, se produjo un aumento del agua disponible o agua azul, capaz de incrementar el recurso hídrico de ecosistemas adyacentes.

Tabla 3. Cuantificación de los elementos del ciclo hidrológico en las parcelas control (C) y tratamiento (T). Pp: precipitación. Tsc: trascolación. Tr: transpiración. Esc: escorrentía superficial.

\begin{tabular}{|c|c|c|c|c|c|}
\hline & Pp & Tsc & Tr & Esc & Disponible \\
\hline C (mm) & 587.3 & 443.9 & 360.2 & 1.9 & 92.5 \\
\hline T (mm) & 587.3 & 438.1 & 343.0 & 2.1 & 104.4 \\
\hline C (\% de la lluvia) & 100 & 75.5 & 61.3 & 0.3 & 15.7 \\
\hline T (\% de la lluvia) & 100 & 74.5 & 58.4 & 0.4 & 17.8 \\
\hline
\end{tabular}

\subsection{Ciclo biogeoquímico}

El aporte de nutrientes a través de la lluvia mostró cambios significativos como consecuencia del MFA. El tratamiento redujo significativamente $(\mathrm{p} \leq 0.05)$ el aporte de K, N y P a través de la escorrentía cortical, e incrementó la exportación de P y K a través de la escorrentía superficial (ver Tabla 4).

Tabla 4. Cuantificación del fósforo $(\mathrm{P})$, nitrógeno $(\mathrm{N})$ y potasio $(\mathrm{K})$ en la precipitación y la escorrentía superficial de las parcelas control y tratamiento.

\begin{tabular}{|c|c|c|c|}
\hline & Precipitación & CONTROL & TRATAMIENTO \\
\hline $\mathrm{P}\left(\mathrm{mg} \mathrm{m}^{-2}\right)$ & $350.0 \pm 642.2$ & $0.001 \pm 0.003$ & $0.002 \pm 0.006$ \\
\hline $\mathrm{N}\left(\mathrm{mg} \mathrm{m}^{-2}\right)$ & $9.8 \pm 17.8$ & $0.03 \pm 0.04$ & $0.03 \pm 0.04$ \\
\hline${\mathrm{K}\left(\mathrm{mg} \mathrm{m}^{-2}\right)}$ & $17.8 \pm 41.2$ & $0.04 \pm 0.04$ & $0.11 \pm 0.11$ \\
\hline
\end{tabular}

De la misma forma, se registró un efecto significativo ( $\mathrm{p} \leq 0.05)$ del MFA en la removilización de los nutrientes en la planta. El contenido de N, C y P tanto en la hojarasca como en las hojas viejas del tratamiento es significativamente $(\mathrm{p} \leq 0.05)$ superior al de la parcela control. De esta forma, parece que el MFA induce una disminución en la eficiencia en el uso de los nutrientes.

\section{Discusión}

El MFA aplicado produjo un incremento en el agua disponible o agua azul, capaz de incrementar el recurso hídrico de ecosistemas adyacentes. Sin embargo, no se registró cambio significativo $(\mathrm{p} \geq 0.05)$ en la capacidad de interceptación de la lluvia así como tampoco en la generación de escorrentía superficial. Esta ausencia de efecto se 
debe probablemente a la estructura aparasolada de la masa, en la cual, a pesar de haber eliminado más de la mitad de los pies iniciales, la masa restante todavía presenta una elevada fracción de cabida cubierta. Por tanto, el incremento de agua disponible se debe probablemente a la disminución del consumo de agua.

El ciclo biogeoquímico global no parece verse significativamente $(\mathrm{p} \geq 0.05)$ afectado por el MFA. Sin embargo, sí se registraron variaciones parciales en la entrada y salida de nutrientes a través del agua. Concretamente, la aplicación de MFA produjo una disminución del aporte de nutrientes a través de la escorrentía cortical. Dicha disminución se debe probablemente a la reducción del número de árboles en la parcela tratamiento. Por otro lado, el MFA parece incrementar la exportación de P y K a través de la escorrentía superficial. Ello puede ser debido al ligero incremento de la escorrentía superficial así como a la presencia del material vegetal triturado.

Por el contrario, el MFA sí parece afectar directamente en el uso de los nutrientes de la planta, reduciendo significativamente $(\mathrm{p} \leq 0.05)$ su eficiencia. El mayor contenido de N, P y K tanto en las hojas como en la hojarasca de la parcela tratamiento, indica probablemente una menor necesidad de recuperar dichos nutrientes justo antes de la caída de la hoja. Probablemente, la mayor disponibilidad de agua de la planta generada por la reducción de la cubierta vegetal reduce la necesidad de almacenar nutrientes en hojas y tallos.

\section{Conclusiones}

El MFA genera un incremento del agua disponible o agua azul, capaz de incrementar el recurso hídrico de ecosistemas adyacentes. Además, dicho incremento no supone un aumento significativo de la escorrentía superficial, y por tanto tampoco de erosión de la suelo.

El consumo de agua por parcela se ve significativamente reducido tras el MFA. Sin embargo, el consumo de agua por individúo incrementa como consecuencia de la disminución de la competencia por el recurso. Dicho incremento probablemente supone también un aumento en el ritmo de crecimiento de los árboles.

El ciclo biogeoquímico asociado al agua no se ve prácticamente afectado por el MFA. Por tanto, se puede afirmar que el manejo aplicado, a pesar de mejorar las condiciones hidrológicas y nutricionales de la planta, no supone una pérdida significativa de nutrientes.

El MFA disminuye la necesidad de recuperar nutrientes de las hojas antes de su caída, mejorando por tanto su estado nutricional.

\section{Referencias}

Callegari, G., Ferrari, E., Garf, G., Iovino, F., Veltri, A., 2003. Impact of thinning on the water balance of a catchment in a mediterranean environment. The Forestry Chronicle 79 (2), 301-306. 
Correia, F. N., 1999. Water resources in the mediterranean region. Water Int. 24 (1), 22-30. Cubasch, U., Von Storch, H.,Waszkewitz, J., Zorita, E., 1996. Estimates of climate change in southern europe derived from dynamical climate model output. Climate Res. 7 (2), 129149.

de Jong, C., Lawler, D., Essery, R., Mar. 2009. Mountain hydroclimatology and snow SeasonalityPerspectives on climate impacts, snow seasonality and hydrological change in mountain environments. Hydrol. Process. 23 (7), 955-961.

Giorgi, F., Lionello, P., 2008. Climate change projections for the mediterranean region. Global Planet. Change 63 (23), 90-104.

Ives, J., Messerli, B., Spiess, E. (Eds.), 1997. Mountains of the World: a Global Priority. Messerli, B. and Ives, J.D. (Eds.), Parthenon, New York.

Joffre, R., Rambal, S., 1993. How tree cover influences the water balance of mediterranean rangelands. Ecology 74 (2), 570-582.

Messerli, B., Viviroli, D., Weingartner, R., 2004. Mountains of the world: vulnerable water towers for the 21st century. Ambio, 29-34.

Molina, A., y Del Campo, A. D. 2011. Estimación del Índice de área foliar en pinares de repoblación con LAI-2000 bajo radiación solar directa: relación con variables de inventario e hidrológicas. Forest systems, (1), 108-121.

Molina, A.J. and A.D. del Campo. 2012. The effects of experimental thinning on throughfall and stemflow: A contribution towards hydrology-oriented silviculture in Aleppo pine plantations. For. Ecol. Manag., 807 269:206-213.

Viviroli, D., Weingartner, R., 2004. The hydrological significance of mountains: from regional to global scale. Hydrol. Earth Syst. Sc. 8 (6), 1017-1030. 
